\section{CPAP: enhancing its use}

\author{
Scott G. Williams • Christopher J. Lettieri • \\ Joseph W. Dombrowsky
}

Published online: 9 March 2012

(C) Springer Science+Business Media, LLC (outside the USA) 2012 treated with CPAP have improved 5-year survival compared to those who remain untreated $(96.4 \%$ and $85.5 \%$ respectively) [11].

While the benefits of CPAP are well recognized, acceptance of treatment and adherence with therapy remains problematic. Early prospective adherence studies showed that only $46 \%$ of patients had regular use of CPAP therapy defined by the Medicare criteria of more than $4 \mathrm{~h}$ of use on at least $70 \%$ of days [12]. These early adherence rates using patient report may have been inaccurate, with patients routinely overestimating use by at least $1 \mathrm{~h}$ [13]. Despite advances in CPAP platforms and comfort features, both short and long-term compliance patterns have not significantly improved. Current estimates indicate that 29\%-83\% of patients prescribed CPAP are non-adherent with therapy ( $\leq 4 \mathrm{~h}$ of use per night) [14].

The definition of adherence has varied amongst investigators, making the existing published data difficult to compare. Further, outcomes related to CPAP use are also difficult to fully comprehend as assessments of the therapeutic response are initially related to the absolute and consistent use of CPAP. This begs the question, "What is the right amount of CPAP use?" Weaver and colleagues sought to determine a dose-response relationship between CPAP and Epworth Sleepiness Scale (ESS) scores, Functional Outcomes associated with Sleep Questionnaire (FOSQ) scores and Mean Sleep Latency Test (MSLT) times. The study demonstrated a linear improvement in all measures that had thresholds over which further improvement was not seen. The nightly use thresholds of $4 \mathrm{~h}, 6 \mathrm{~h}$, and $7.5 \mathrm{~h}$ for ESS, MSLT, and FOSQ scores respectively, help us establish desired optimal nightly CPAP use for an individual patient [15]. The authors found that $4 \mathrm{~h}$ per night was associated with improved outcomes. Greater use of CPAP was associated with slightly better outcomes. Publications
S. G. Williams $\cdot$ C. J. Lettieri $(\bowtie) \cdot$ J. W. Dombrowsky

Sleep Disorders Center,

Walter Reed National Military Medical Center,

Building 9a, Arrowhead Zone, 2nd Floor,

8901 Wisconsin Avenue,

Bethesda, MD 20889, USA

e-mail: christopher.lettieri@us.army.mil 
such as this were used to establish $4 \mathrm{~h}$ per night as a minimum threshold for acceptable CPAP use. It should be noted that relatively few patients in these studies used CPAP for $>7 \mathrm{~h}$ per night so there is little data to quantify the incremental improvement that extended use provides. However, given that insufficient sleep has detrimental effects on health and quality of life, the goal of therapy should be the use of CPAP on all nights for 7-8 h per night.

In order to maximize the role of CPAP and continue to decrease the burden of disease associated with OSA, the treating physician must both enhance its ability to ablate obstructive events and improve sleep quality, as well as promote better acceptance of and adherence to therapy. This means improving the ability for CPAP to do what it is intended to do under ideal conditions (efficacy) in addition to improving the real-world application of CPAP therapy (effectiveness). Understanding the common barriers to CPAP use, application of emerging technologies, and strategies to maximize both the therapeutic response and adherence are fundamentally important in the care of patients with OSA.

\section{Patient education, follow-up, and continuity of care}

The etymology of the word "doctor" comes from the Latin docere meaning to show or teach. In an increasingly complex and efficiency-driven medical climate, teaching has become a secondary consideration during many clinical encounters. When it comes to changing habits or behaviors, however, medical professionals must spend as much time as is necessary to ensure that patients are fully informed regarding the risks, benefits, and alternatives of any treatment regimen. A better understanding of the adverse impact of untreated OSA and the potential benefits of CPAP therapy is perhaps the most important, but often overlooked, aspect in long-term adherence. Advancements in PAP platforms, the addition of comfort features or improved mask interfaces will have little impact if the patient does not understand why OSA should be treated. This was highlighted by Rose and colleagues who described the link between the patient's knowledge of the health risk of untreated OSA and CPAP compliance [16].

A competent and caring primary clinician sets the stage for an optimal experience with CPAP [17]. As soon as OSA is suspected, the primary clinician must discuss the reasons for referral for overnight polysomnography as well as the treatment options if OSA is discovered. After diagnosis, several different educational modalities have been proposed to optimize the use of CPAP. These include repeated home visits, involvement of spouses, telephone follow-up assessments, video supplements, and intensive patient educational programs [18•]. A comprehensive, intensive educational approach appears to increase patient understanding and awareness, facilitate acceptance of therapy, and improve adherence [19-21].

While intensive education may benefit individual patients, the clinical improvement that it generates may not outweigh the allocation of resources it requires. Given the increasing demand for sleep evaluations, intensive educational programs may not be widely available. Therefore, the cost-effectiveness of these programs remains controversial $[22,23]$. Although intensive programs may remain out of reach for some patients, even simple efforts can be beneficial [24•]. Ensuring that patients understand why OSA needs to be treated and the adverse effects it has on both health and quality of life can promote better acceptance of therapy. Understanding the proper use and maintenance of CPAP can also prevent simple problems from threatening continued treatment especially during the initial adjustment period of CPAP. Sadly, CPAP is still associated with an unfavorable social stigma and may cause embarrassment for some patients. This often limits both acceptance of and continued use of CPAP [25]. Patients may feel ashamed to wear a mask in front of their bed partner, particularly if they are younger or are not in a committed, long-term relationship [16].

Recent publications have demonstrated that physiciandirected, multi-disciplinary education during the initiation of CPAP therapy may improve therapeutic adherence to therapy and clinical outcomes [26, 27]. Group educational programs and CPAP support groups provide a social network of other patients with similar concerns. They can assist each other to overcome psychosocial complaints. For those who have a consistent bed partner, involving them in the treatment process and allowing them to participate in these clinical encounters may encourage a discussion about the "perceived" versus "real" aesthetic impact of the device and mitigate any self-consciousness that exists. Involving the partner in the discussion about the long-term health impact of untreated OSA may also potentiate buy-in. Group educational programs have the additional benefit of incorporating several patients at the same time, which can improve the efficiency of sleep clinics and increase access to care.

Follow-up assessments and continuity of care are essential to encourage continued use of CPAP, optimize the therapeutic response, achieve better outcomes, and identify potential barriers to effective therapy. The first few days to weeks of CPAP use is highly predictive of long-term adherence. In a study of 100 patients with moderate to severe OSA, $84 \%$ of patients who used CPAP for greater than $4 \mathrm{~h}$ per night on the third day of treatment continued to have regular use of CPAP at day 30. In contrast, among those who used CPAP for less than $4 \mathrm{~h}$ per night at day 3, only $26 \%$ were regular users of CPAP at day 30 [28]. Assessments shortly after initiating CPAP can have a profound 
effect on continued use. The initial transition to CPAP therapy can be associated with several, often easily resolved barriers. These are primarily related to the mask interface and comfort with the delivered pressure. If patients can overcome the change to their bedtime ritual, they adapt to CPAP after a short period of time. However, failure to recognize and resolve these common barriers may lead to frustration, discomfort, and abandonment of therapy. Therefore, it is vital to have an integrated system of care that is prepared to respond to patient issues and concerns particularly during the first few weeks of use [29]. Even a brief telephone follow-up during the first week of CPAP use and a clinic evaluation after the first month can make the difference between successful treatment with long-term adherence and discontinuation of CPAP. Having a close working relationship with the patient's durable medical equipment (DME) vendor is critical. These vendors can often handle the most common CPAP-related questions, troubleshoot problems with mask interfaces, and mitigate the need for unscheduled clinical evaluations.

\section{Recognizing and overcoming common barriers to CPAP therapy}

Numerous patient, mask, and pressure-related variables can negatively impact the tolerance and effectiveness of CPAP. Familiarity with the most common problems encountered with CPAP will allow clinicians to make quick, often simple interventions. Early recognition and implementation of measures to overcome these barriers may increase the confidence of the patient, foster better adherence, or prevent discontinuation of CPAP.

\section{Insomnia}

Insomnia is common in patients with OSA and these disorders are frequently considered to be comorbid. Several reports have found that insomnia is present in $39 \%-58 \%$ of patients with OSA. Resolution of sleep-disordered breathing often leads to a resolution of insomnia. Unfortunately, the concurrence of insomnia in patients with OSA has an adverse impact on the use of CPAP, especially in the initial treatment period. Transitioning to CPAP often causes difficulties initiating sleep. Exacerbation of pre-existing insomnia may lead patients to abandon CPAP altogether. This was highlighted by Wickwire and associates who observed that complaints of sleep maintenance insomnia predicted poor CPAP adherence [30]. Treatment of insomnia during the initial phase of OSA therapy can enhance tolerance, effectiveness, and increase patient satisfaction [31]. Common insomnia treatments include cognitive behavioral therapy or soporific medication. For select patients with comorbid insomnia and OSA, a daytime nap in the sleep clinic combined with guided imagery and relaxation techniques has been shown to improve adherence [32].

\section{Claustrophobia}

Anxiety or claustrophobia can be an important barrier to CPAP therapy [33]. For some patients, CPAP may unmask latent anxiety and contribute to treatment refusal. However, several strategies can be very effective and most patients with claustrophobia can achieve successful treatment with CPAP. Selecting a comfortable mask interface which minimizes feelings of confinement is important. Interestingly, smaller masks such as nasal pillows may not always accomplish this goal, and many patients may find full face masks or even a total face mask more palatable. As such, mask selection should be individualized for each patient. Desensitization to both the mask and the delivered pressure is accomplished through a variety of different protocols. While the specific therapeutic methods are beyond the scope of this article, they share the common feature of progressive daytime use and graduated nocturnal use until the patient is comfortable wearing CPAP throughout the night. Incorporating a behavioral sleep medicine (BSM) specialist in the care of those patients who have ongoing difficulties is frequently beneficial [34].

\section{Chronic rhinitis}

Chronic sinus congestion affects approximately $40 \%$ of patients with OSA. There are several potential etiologies for this common association including vasomotor rhinitis with upper airway edema caused by increased upper airway pressure differentials, the vibratory impact of snoring, and nocturnal gastroesophageal reflux. Regardless of the cause, the presence of nasal congestion can have a tremendous impact on the tolerance and effectiveness of CPAP.

In order for nasal CPAP to work, it must overcome the resistance in the nasopharynx. Patients with chronic sinus congestion may require higher pressures or the nasal airflow may not adequately stent the hypopharynx or oropharynx. The use of heated humidification can significantly decrease the impact of rhinitis in patients on CPAP and has largely eliminated the development of acute, transient sinus congestion commonly seen during the initial treatment period. In a study by Koutsourelakis and colleagues, heated humidification reduced nasal symptoms, nasal resistance, and inflammatory cell infiltration of the nasal mucosa [35].

When present, chronic rhinitis should be treated prior to initiation of CPAP. Intranasal steroids are often effective, but their use should be limited to those with sinus congestion significant enough to impair the use of CPAP. In unselected patients, there is little evidence that nasal corticosteroids 
improve CPAP tolerability. In a study by Strobel and coworkers, twice daily fluticasone used for 10 days prior to and 4 weeks following the initiation of CPAP provided no improvement in nasal symptoms, nasal peak flow values, or CPAP use [36]. Similarly, Ryan and colleagues found that humidification, but not fluticasone, decreased the frequency of nasal symptoms in patients on CPAP. In addition, the authors did not observe any improvement in CPAP adherence with fluticasone [37].

Surgical options may provide some adjunctive benefit for patients suffering from unresolved treatment-limiting sinus congestion despite aggressive medical therapy. In a small, pilot trial, turbinate reduction lead to subjective improvements in nasal obstruction among patients treated with CPAP [38].

\section{Sleep fragmentation}

Sleep fragmentation is common in patients with OSA. Unconscious mask removal may signal nocturnal arousals in those who are unaware of any awakenings. There are several common causes of continued sleep disruption despite CPAP therapy. Obviously not all are the result of CPAP or sleepdisordered breathing. However, when present, it should be addressed as persistent nocturnal awakenings can lead to continued sleepiness, anxiety, or cause patients to lose confidence in CPAP.

Sleep fragmentation, especially early morning awakenings, can be a clue that the patient has incompletely treated OSA. Adjusting the CPAP pressure is often all that is required. Most PAP platforms will report the residual AHI on therapy. An elevated value suggests that the patient is not receiving an adequate delivered pressure. This may be the result of mask leak or mouth breathing. If these are not clinically suspected, the patient likely needs a higher prescribed pressure. For patients using a fixed CPAP device, empirically increasing their pressure by $2 \mathrm{~cm} \mathrm{H} 2 \mathrm{O}$ and reassessing clinically may be effective. In those treated with an auto-adjustable (APAP) device, the lower limit of the pressure range should be set at the $90 \%-95 \%$ pressure (pressure in which the patient spends $90 \%$ or $95 \%$ of their total sleep time). The upper limit should be set at $2-4 \mathrm{~cm} \mathrm{H} 2 \mathrm{O}$ above the lower limit. While these empiric adjustments avoid the need for additional testing, clinicians should be cautioned that the increased pressures may precipitate central apneic events or contribute to further mask leakage or patient intolerance. If adjusting the pressure is unsuccessful the patient should be re-evaluated in the sleep lab.

Many CPAP devices have an alarm feature that can be activated when the mask is removed. As the patient adjusts to the device they will gradually become conditioned to wake up when the mask is removed so they can put it back on. Over time, non-deliberate mask removal tends to decrease. Reassuring the patient that slow, steady progress is the norm will minimize the feeling of "failure" which ultimately leads to discontinuation of therapy.

\section{Mask leaks}

The first step to preventing problems with the mask interface is proper fit. The number of masks is rapidly increasing, and most clinicians cannot keep up with the latest technological advances. DME vendors should educate patients and provide a formal mask fitting by a trained therapist. With few exceptions the choice should be primarily influenced by the patient's comfort and wishes. However, facial structure and facial hair play an important part of mask selection and a good respiratory therapist can identify potential problems before the patient begins therapy. Prescribed pressures can also limit the range of effective masks. Typically nasal pillows are less effective with higher delivered pressures and many clinicians limit their use to pressures less than $12 \mathrm{~cm} \mathrm{H} 2 \mathrm{O}$. Habitual mouth breathing and chronic sinus congestion can limit the effectiveness of nasal masks and often full face masks are recommended.

Even with a proper fit, mask leak is a common complaint, especially during the early stages of CPAP therapy. Dry eyes, conjunctivitis, or loud noises from the mask are clues the mask is improperly sealed. If the mask does not fit properly, patients may quickly lose interest in the therapy particularly if they do not experience improvements in daytime symptoms. During the initial educational visit, the clinician must reassure the patient that excessive mask leak is not normal and can easily be resolved. Changing the size or type of mask may be necessary and the patient should contact their DME vendor to re-fit their mask. If a patient has significant facial hair, nasal pillows may provide a better seal. Additionally, some simple behavioral interventions can engrain sleep habits to minimize the possibility the mask will dislodge throughout the night. One example is a muscle memory exercise whereby the patient places their hand over the mask whenever they move while awake. Over time, this becomes a reflexive behavior that continues unconsciously during sleep. It is important to inform the patient that they should turn on the CPAP at full pressure (not using the ramp feature) prior to donning the mask in order to improve the seal. Masks should be replaced every 3-6 months as older masks may experience silicone breakdown and begin to leak.

Nasal or oral dryness are other common adverse effects of CPAP. Ensuring that patients are properly using the humidifier may mitigate these problems. Significant dry mouth typically suggests mouth breathing. When present, consider switching to a full face mask or adding a chin strap to a nasal mask. 


\section{Other common barriers}

Gastric distention from swallowing air is a common complaint with CPAP. Flexion of the neck (from using too many pillows) or untreated sinus congestion are common causes. Slightly hyper extending the neck by placing a rolled-up towel under the lower cervical spine can also minimize air swallowing. If gastric distention persists, consider decreasing the pressure. In this case, the clinician must balance the relative impact of incompletely treated OSA with patient comfort.

Pressure ulceration or skin breakdown is never normal and should be addressed. Replacing the mask at normal intervals and ensuring it is regularly cleaned may be all that is necessary to prevent these problems. Changing the mask type or size may be required. In additional, silicone pads and commercially available skin barriers and creams may help prevent the development of mask-induced skin breakdown.

Excessive noise from the CPAP device or mask is also abnormal. Newer masks and CPAP devices have been designed to produce very little noise, often less than 35 decibels. Loud noises are usually due to improper assembly of the CPAP, mask or tubing and patients should be referred to their DME supplier or bring their machine into the clinic.

Condensation in the mask may be due to a temperature or humidity difference between the ambient air and the inhaled air. Newer CPAP devices can selfadjust their temperature and humidity to prevent this. Insulation around mask tubing can be helpful. Another strategy would be to increase the ambient temperature of the room, especially during the winter. This can aid in decreasing the temperature gradient which prevents the formation of condensation.

Residual sleepiness or fatigue may be present despite adequate CPAP use. Continued daytime symptoms may lead patients to lose confidence in CPAP or discourage them from continued use. For patients reporting persistent sleepiness, there are several strategies that clinicians should explore. First, ensure that the CPAP pressures are effective by interrogating the therapy data and inquiring about residual snoring. Second, make certain the patient is obtaining an adequate amount of sleep. Third, identify comorbid conditions, medications, or other potential causes of sleep disruption that could be causing or contributing to daytime sleepiness. If sleepiness continues despite adherence with CPAP or some other identifiable factor, the use of a wake-promoting agent should be considered.

If the patient is still having difficulty with CPAP compliance, it may be necessary to perform a repeat sleep evaluation or refer them to a sleep physician.

\section{PAP platforms and comfort features}

\section{PAP platforms}

Several different PAP platforms are available for the treatment of OSA. CPAP delivers a fixed pressure and has been the treatment of choice for OSA for the past three decades. However, newer modalities have emerged which may offer greater patient comfort, more effective therapy, and improved adherence.

BiPAP delivers one pressure during the inspiratory phase and a lower pressure during the expiratory phase. BiPAP was previously used as rescue for CPAP intolerance, with the hypothesis that this mode would improve patient comfort with fewer leaks. However, this has largely been replaced by newer expiratory pressure relief comfort features. Currently, there is a limited role for BiPAP in the treatment of OSA. It can be considered for patients requiring pressures higher than those offered by standard CPAP platforms or those who cannot tolerate maximal CPAP pressures.

Both the severity of OSA and the pressure required to ablate events may vary between nights, body position, and stage of sleep. As such, fixed CPAP pressures may deliver too much pressure at certain times and insufficient pressures at others. Auto-adjustable/auto-titrating positive airway pressure (APAP) devices deliver a variable pressure throughout the night in response to airflow limitations. These devices allow delivery of a minimally effective pressure which can lead to an overall reduced mean nightly pressure. In theory, this could improve both patient comfort and PAP acceptance. However, objective improvements in adherence with APAP therapy compared to standard CPAP has been variable and no clear advantage in efficacy or subjective sleepiness has been observed [39•]. In a metaanalysis, APAP was associated with increased patient preference but no improvement in quality of life or daytime symptoms. Some studies have shown an increase in PAP usage, but the absolute change is typically less than $30 \mathrm{~min}$ per night [40].

\section{Heated humidification}

Heated humidifiers for CPAP were developed in order to increase the comfort for those patients who complained of excessive nasal or oral dryness. Most current PAP devices now include an integrated heated humidification system. Heated humidity delivers greater moisture than cool humidification. It may be particularly useful for those with mouth or mask leaks complaining of dry oral or nasal mucosa and nasal congestion. The level of humidity can be adjusted by the patient depending on environmental and seasonal changes. An initial crossover trial comparing heated humidity, cold 
passover humidity, and a washout period without humidity demonstrated enhanced CPAP compliance when heated humidified air is used [41]. Unfortunately, other investigators have not confirmed that this comfort measure leads to improved compliance [34, 42].

\section{Pressure comfort settings}

Expiratory pressure relief, or flexible CPAP, is a recent feature of PAP that was developed to improve patient comfort by allowing the airway pressure to fall below the prescribed pressure during expiration. However, current data is mixed regarding improved PAP adherence. A Cochrane database review of pressure modification to improve comfort and compliance demonstrated that while patients preferred these comfort settings, CPAP use did not improve [43]. The reviewers commented that across all of these studies, there was a high level of use in the control groups, which may dilute any "real-world" effect of the comfort features [18]. It is important to note, however, that using these features may lead to more sleep fragmentation, more noise from the device, and a risk of inadequate therapy due to reduced end-expiratory pressure.

\section{Ramp feature}

Most PAP devices offer a ramp feature which provides an on-demand, temporary reduction in the delivered pressure. The pressure gradually increases over approximately 20 min, allowing patients to fall asleep prior to reaching the higher prescribed pressure. Similar to the expiratory pressure relief, there is no data to show that this improves adherence [18]. Additionally, the ramp feature may fragment sleep as it delivers an inadequate pressure for the first 15-20 min of sleep. As with all comfort features, the benefits to patient comfort and continuation of therapy must be weighed against the inherent costs and potential risks.

\section{Improving the initial experiences with CPAP}

The use of CPAP during the initial treatment period has been consistently shown to predict long-term use. Further, longterm adherence patterns may be determined within the first few days of therapy $[28,44]$. As such, strategies aimed at improving the initial experiences with CPAP have been the focus of a number of recent studies.

For select patients, the use of non-benzodiazepine sedative hypnotic medications (BZRAs) can be a useful adjunct. A retrospective review found that the use of zolpidem or eszopiclone on the night of in-lab titration is predictive of improved CPAP compliance. During the first month of therapy, $77 \%$ of patients who had used a BZRA met the Medicare definition for regular use of CPAP compared with only $57.6 \%$ among patients who did not use a sleep aid [45]. A validating, randomized, double-blind, placebo-controlled prospective study by the same group found that among those premedicated with eszopiclone during the CPAP titration, CPAP was used on more nights $(75.9 \% \pm 20.0 \%$ vs $60.1 \% \pm 24.3 \%$; $P=0.005)$ and for more hours per night $(4.8 \pm 1.5$ vs $3.9 \pm 1.8$; $P=0.03$ ) [46]. Zolpidem has also been shown to improve the quality of overnight polysomnography and decrease the need to repeat the study [47].

Recent data have shown that eszopiclone use during the first 2 weeks of CPAP therapy can also improve adherence rates. A randomized, placebo-controlled trial demonstrated that those receiving a 2 -week course of eszopiclone used CPAP for $20.8 \%$ more nights and 1.1 more hours per night during the first 6 months of therapy [48•]. However, in a similar trial using zolpidem, Bradshaw and colleagues did not find the same improvement in CPAP compliance with sedative therapy [49]. In addition, ramelteon has been shown to improve insomnia symptoms but it was not associated with increased APAP adherence [50]. Overall, however, sedative medications during the diagnostic and initial treatment phase are safe and do not appear to alter the sleep architecture or severity of sleep disordered breathing and may lead to an improved initial experience with CPAP and subsequently better adherence with therapy.

\section{Conclusions}

CPAP remains a safe and very effective means to improve sleep-disordered breathing, and the rapid advances in technology have resulted in a simple-to-use, portable and unobtrusive machine. Despite advances in CPAP platforms and comfort features, CPAP remains underutilized largely because of poor patient acceptance. Adequate education and rapid troubleshooting of initiation problems is critical to maximizing CPAP use.

Although the quality of the initial phase of therapy is extremely important to allow patients to experience the full benefit of PAP therapy, appropriate follow-up is critical to ensure continued resolution of sleep-disordered breathing and improved outcomes. Patients should be re-evaluated after the initial month of therapy. It is recommended that patients communicate with the home health care agency or DME vendor at least every 3-6 months in order to replace the mask and tubing. Each interaction is an opportunity to assess for any residual symptoms or equipment issues. The patients should follow-up with their primary physician or a sleep disorders center annually to determine if the pressure settings are correct, to verify that the device is working properly, and to ensure that the treatment response and outcomes are optimized. 
Disclosure S. G. Williams: none; C. J. Lettieri: speakers' bureaus for Cephalon and Sunovion; J. W. Dombrowsky: none.

\section{References}

Papers of particular interest, published recently, have been highlighted as:

- Of importance

1. Nieto FJ, Young TB, Lind BK, et al. Association of sleep disordered breathing, sleep apnea, and hypertension in a large community-based study: Sleep Heart Health Study. JAMA. 2000;283:1829-36.

2. Shepard Jr JW. Hypertension, cardiac arrhythmias, myocardial infarction, and stroke in relation to obstructive sleep apnea. Clin Chest Med. 1992;13:437-58.

3. Drager LF, Bortolotto LA, Figueiredo AC, et al. Effects of continuous positive airway pressure on early signs of atherosclerosis in obstructive sleep apnea. Am J Respir Crit Care Med. 2007;176:706-12.

4. Gami AS, Hodge DO, Herges RM, et al. Obstructive sleep apnea, obesity, and the risk of incident atrial fibrillation. J Am Coll Cardiol. 2007;49:565-71.

5. Punjabi NM, Shahar E, Redline S, et al. Sleep Heart Health Study Investigators. Sleep-disordered breathing, glucose intolerance, and insulin resistance: the Sleep Heart Health Study. Am J Epidemiol. 2004;160(6):521-30.

6. Shahar E, Whitney CW, Redline S, et al. Sleep-disordered breathing and cardiovascular disease: cross-sectional results of the Sleep Heart Health Study. Am J Respir Crit Care Med. 2001;163(1):19-25.

7. Kushida CA, Littner MR, Hirshkowitz M, et al. Practice parameters for the use of continuous and bilevel positive airway pressure devices to treat adult patients with sleep-related breathing disorders. Sleep. 2006;29(3):157-71.

8. Montserrat JM, Ferrer M, Hernandez L, et al. Effectiveness of CPAP treatment in daytime function in sleep apnea syndrome: a randomized controlled study with an optimized placebo. Am J Respir Crit Care Med. 2001;164(4):608-13.

9. Taskin U, Yigit O, Acioglu E, et al. Erectile dysfunction in severe sleep apnea patients and response to CPAP. Int J Impot Res. 2010;22:134-9.

10. Sanchez AI, Buela-Casal G, Bermudez MP, Casas-Maldonado F. The effects of continuous positive airway pressure treatment on anxiety and depression levels in apnea patients. Psychiatry Clin Neurosci. 2001;55(6):641-6.

11. Campos-Rodriguez F, Pena-Grinan N, Reyes-Nunez N, et al. Mortality in obstructive sleep apnea-hypopnea patients treated with positive airway pressure. Chest. 2005;128:624-33.

12. Kribbs NB, Pack AI, Kline LR, et al. Objective measurement of patterns of nasal CPAP use by patients with obstructive sleep apnea. Am Rev Respir Dis. 1993;147:887-95.

13. Engleman HM, Asgari-Jirandeh N, McLeod AL, Ramsay CF, Deary IJ, Douglas NJ. Self-reported use of CPAP and benefits of CPAP therapy. Chest. 1996;109:1470-6.

14. Pruitt B: Top 10 Practices to Increase CPAP Compliance. RT for Decision Makers in Respiratory Care 2009, 22(2).

15. Weaver TE, Maislin G, Dinges DF, Bloxham T, George CFP, Greenberg $\mathrm{H}$, et al. Relationship between hours of CPAP use and achieving normal levels of sleepiness and daily functioning. Sleep. 2007;30:711-9.

16. Rose MW. Positive airway pressure adherence: problems and interventions. Sleep Med Clin. 2006;1(4):533-39.

17. Zoidis JD: Improving compliance with CPAP. RT for Decision Makers in Respiratory Care 2007, 20(6).

18. - Smith I, Nadig V, Lasserson TJ. Educational, supportive and behavioural interventions to improve usage of continuous positive airway pressure machines for adults with obstructive sleep apnoea. Cochrane Database Syst Rev. 2009;4:CD007736. Education regarding the adverse impact of untreated OSA and the potential benefits of CPAP therapy is critical to promoting better outcomes and improved long-term adherence. This review explores the benefits of a variety of different educational modalities and observed that while intensive educational programs were very beneficial, even simple strategies improved adherence.

19. Golay A, Girard A, Grandin S, et al. A new educational program for patients suffering from sleep apnea syndrome. Patient Education and Counseling. 2006;60:220-7.

20. Likar LL, Panciera TM, Erickson AD, Rounds S. Group education sessions and compliance with nasal CPAP therapy. Chest. 1997;111:1273-7.

21. Hoy CJ, Vennelle M, Kingshott RN, et al. Can intensive support improve continuous positive airway pressure use in patients with the sleep apnea/hypopnea syndrome? Am J Respir Crit Care Med. 1999;159:1096-100.

22. Hui DS, Chan JK, Choy DK, et al. Effects of augmented continuous positive airway pressure education and support on compliance and outcome in a Chinese population. Chest. 2000;117:1410-6.

23. Meurice JC, Ingrand $P$, Portier F, et al. A multicentre trial of education strategies at CPAP induction in the treatment of severe sleep apnoea-hypopnoea syndrome. Sleep Medicine. 2007;8:3742.

24. • Chervin RD, Theut S, Bassetti C, Aldrich MS. Compliance with nasal CPAP can be improved by simple interventions. Sleep 1997;20(4):284-9. In order to optimize the effect and use of CPAP, it is important to understand the potential barriers that impair acceptance of and adherence with therapy. This review explores psychosocial and other common factors that adversely affect adherence and outcomes related to CPAP.

25. Shapiro GK, Shapiro CM. Factors that influence CPAP adherence: an overview. Sleep Breath. 2010;14(4):323-35.

26. Golay A, Girard A, Grandin S, Metrailler J-C, Victorion M, Lebas $\mathrm{P}$, et al. A new educational program for patients suffering from sleep apnea syndrome. Patient Educ Couns. 2006;60:220-7.

27. Walter RJ, Shah AA, Lettieri CJ. The effects of individual vs. group education on continuous positive airway pressure compliance in patients with obstructive sleep apnea. Chest. 2010;138:889A.

28. Budhiraja R, Parthasarathy S, Drake CL. Early CPAP use identifies subsequent adherence to CPAP therapy. Sleep. 2007;30:320-4.

29. Chowdhuri S. Continuous positive airway pressure for the treatment of sleep apnea. Otolaryngol Clin North Am. 2007;40:80727.

30. Wickwire EM, Smith MT, Birnbaum S, Collop NA. Sleep maintenance insomnia complaints predict poor CPAP adherence: a clinical case series. Sleep Med. 2010;11:772-6.

31. Luyster FS, Buysse DJ, Strollo PJ. Comorbid insomnia and obstructive sleep apnea: challenges for clinical practice and research. J Clin Sleep Med. 2010;6:196-204.

32. Krakow B, Ulibarri V, Melendrez D, et al. A daytime, abbreviated cardio-respiratory sleep study to acclimate insomnia patients with sleep disordered breathing to positive airway pressure (PAP-NAP). J Clin Sleep Med. 2008;4(3):212-22.

33. Chasens ER, Pack AI, Maislin G, et al. Claustrophobia and adherence to CPAP treatment. West J Nurs Res. 2005;27(3):307-21.

34. Haynes PL. The role of behavioral sleep medicine in the assessment and treatment of sleep disordered breathing. Clin Psych Rev. 2005;673-705.

35. Koutsourelakis I, Vagiakis E, Perraki E, et al. Nasal inflammation in sleep apnoea patients using CPAP and effect of heated humidification. Eur Respir J. 2011;37:587-94.

36. Strobel W, Schlageter M, Andersson M, et al. Topical nasal steroid treatment does not improve CPAP compliance in unselected patients with OSAS. Respir Med. 2011;105(2):310-5. 
37. Ryan S, Doherty LS, Nolan GM, McNicholas WT. Effects of heated humidification and topical steroids on compliance, nasal symptoms, and quality of life in patients with obstructive sleep apnea syndrome using nasal continuous positive airway pressure. $\mathrm{J}$ Clin Sleep Med. 2009;15(5):422-7.

38. Powell NB, Zonato AI, Weaver EM, et al. Radiofrequency treatment of turbinate hypertrophy in subjects using continuous positive airway pressure: a randomized, double-blind, placebocontrolled clinical pilot trial. Laryngoscope. 2001;111(10):178390 .

39. • Smith I, Lasserson TJ. Pressure modification for improving usage of continuous positive airway pressure machines in adults with obstructive sleep apnoea. Cochrane Database Syst Rev. 2009;4: CD003531. Several advances in PAP platforms and comfort features have been widely implemented in an attempt to improve patients' comfort with CPAP and, ultimately, increase adherence and therapy. This Cochrane database review of pressure modification demonstrated that while patients preferred these comfort features, they did not improve CPAP use.

40. Ayas NT, Patel SR, Malhotra A, et al. Auto-titrating versus standard continuous positive airway pressure for the treatment of obstructive sleep apnea: results of a meta-analysis. Sleep. 2004;27:249-53.

41. Massie CA, Hart RW, Peralez K, Rishards GN. Effects of humidification on nasal symptoms and compliance in sleep apnea patients using continuous positive airway pressure. Chest. 1999;116(2):403-8

42. Mador MJ, Krauza M, Pervez A, et al. Effect of heated humidification on compliance and quality of life in patients with sleep apnea using nasal continuous positive airway pressure. Chest. 2005;128(4):2151-8.
43. Bakker JP, Marshall NS. Flexible pressure delivery modification of continuous positive airway pressure for obstructive sleep apnea does not improve compliance with therapy: systematic review and meta-analysis. Chest. 2011;139(6):1322-30.

44. Drake CL, Day R, Hudgel D, et al. Sleep during titration predicts continuous positive airway pressure compliance. Sleep. 2003;26:30811.

45. Collen J, Lettieri C, Kelly W, Roop S. Clinical and polysomnographic predictors of short-term continuous positive airway pressure compliance. Chest. 2009;135:704-9.

46. Lettieri CJ, Collen JF, Eliasson AH, Quast TM. Sedative use during continuous positive airway pressure titration improves subsequent compliance: a randomized, double-blind, placebocontrolled trial. Chest. 2009;136:1263-8.

47. Lettieri CJ, Eliasson AH, Andrada T, et al. Does zolpidem enhance the yield of polysomnography? J Clin Sleep Med. 2005;1(2):129-31.

48. - Lettieri CJ, Shah AA, Holley AB, et al. Effects of a short course of eszopiclone on continuous positive airway pressure adherence: a randomized trial. Ann Intern Med. 2009;151:696-702. Long-term patterns of CPAP use are frequently established early and the initial experiences with CPAP can greatly influence the patient's willingness to continue or abandon therapy. This study found that a short course of eszopiclone during the first 2 weeks of therapy improved long-term adherence and continuation of CPAP.

49. Bradshaw DA, Ruff GA, Murphy DP. An oral hypnotic medication does not improve continuous positive airway pressure compliance in men with obstructive sleep apnea. Chest. 2006;130:1369-76.

50. Gooneratne NS, Gehrman P, Gurubhagavatula I, et al. Effectiveness of ramelteon for insomnia symptoms in older adults with obstructive sleep apnea: a randomized placebo-controlled pilot study. J Clin Sleep Med. 2010;6(6):572-80. 